\title{
Research on TD-LTE wireless communication network propagation model optimization based on visual simulation and GIS
}

\author{
Tongyan Li', Shuchun Hua², Le Kang ${ }^{3^{*}}$ (1) and Sheng-Hung Chang ${ }^{3}$
}

\author{
*Correspondence: \\ kangle@hbue.edu.cn \\ ${ }^{3}$ School of Business \\ Administration, Hubei \\ University of Economic, \\ Wuhan, China \\ Full list of author information \\ is available at the end of the \\ article
}

\begin{abstract}
With the support of GIS spatial analysis technology, based on an in-depth study of the wireless propagation environment of a city, combined with the analysis of project requirements, it proposes to use the SPM model to correct the propagation model parameters, using SPM. The wireless propagation model, and research and analysis of the SPM wireless propagation model correction algorithm, further corrected the parameters of a city's SPM wireless propagation model. On this basis, the propagation loss of several classic propagation models in different environments is compared, and the SPM propagation model suitable for the signal frequency band and propagation environment of this study is selected. The correction of the SPM propagation model is based on the designed correction principle and correction process, that is, the weighted least square method is used to fit and analyze the measured level data to obtain an SPM prediction improvement model with local characteristics, and according to the designed verification link. Evaluation of the correction results shows that the accuracy requirements are met. Based on the corrected SPM prediction model, link loss calculations were performed on the 13 test base stations studied in the experiment, and the effective coverage radius of each base station community was obtained. In combination with GIS technology, model parameters and workers of each base station participated in the electronic map loading of the area Go to the network planning software to get the wireless signal coverage prediction map of each base station. Finally, according to the technical requirements of the TD-LTE system network planning and network optimization engineering, the objectiveness and rationality of the site selection and number of base stations in the area were verified, and specific problems regarding poor coverage and overlapping coverage in the area were proposed.
\end{abstract}

Keywords: TD-LTE, Propagation model, Path loss, Coverage prediction, Network optimization spalling

\section{Introduction}

This thesis takes TD-LTE as the research object. TD-LTE is now the most used network service, but because the technology is independently developed in China, there is no mature communication network construction experience to learn from, so TDLTE wireless network Planning is a challenge. The first step of communication network author(s) and the source, provide a link to the Creative Commons licence, and indicate if changes were made. The images or other third party material in this article are included in the article's Creative Commons licence, unless indicated otherwise in a credit line to the material. If material is not included in the article's Creative Commons licence and your intended use is not permitted by statutory regulation or exceeds the permitted use, you will need to obtain permission directly from the copyright holder. To view a copy of this licence, visit http:// creativecommons.org/licenses/by/4.0/. 
planning is to determine the propagation model of radio wave signal coverage prediction, because the propagation model is suitable to predict the median loss of the signal from transmission to reception. Although domestic and foreign scholars have obtained many classic propagation models through experimental research, and these classic models have certain universality, the environment in specific scenes changes, which will lead to less accurate predictions. Therefore, in network planning, the path loss formula or the propagation model must be corrected to obtain more accurate propagation prediction losses, and preparations are made for subsequent coverage planning, capacity planning, and link budgeting.

In practical applications, GIS-based communication system planning, coverage forecasting, data analysis and processing, network quality monitoring and analysis, and other functions [1-3] are all kinds of communication data with geographic attributes and computer technology. The combination of GIS spatial analysis technology, visualization and simulation technology enables users to easily and intuitively see the coverage effect, scope and obtain technical support for network planning and optimization. The necessity and importance of the introduction of GIS technology from the perspective of actual needs and application prospects [4-6], and the construction of various types of modern dedicated communication information systems based on integrated development with GIS, are provided for the management, analysis, and planning of all communication resources. Brand-new methods and means analyze the actual key problems of communication network resource data and realize the function of visual information management using GIS technology in communication network resource management $[7,8]$ and propose a method using drive test data A model for propagation correction, which can be applied to mobile networks of different standards and standards [9, 10]; according to the existing wireless signal propagation prediction model, using the $\mathrm{CW}$ continuous wave test method, by improving the traditional model algorithm and correction process, Prediction of the signal coverage in a certain propagation environment and estimation of the propagation loss value of a certain distance of the signal have obtained good simulation results. It provides a strong engineering method for accurate coverage prediction of wireless signals in specific research areas [11-13]. During the widespread application of the second-generation mobile communication systems (GSM and CDMA), the research work on propagation models has developed unprecedentedly, and a propagation model has been formed. This propagation model is suitable for many different wireless propagation environments $[14,15]$. According to the different application scenarios of propagation models, they can generally be divided into three categories: macro-cell propagation models, micro-cellular propagation models, and indoor propagation models [16-19]. The application status and trends of GIS technology in the communication industry are analyzed, and the visualization of GIS and the optical fiber routing management and fault location of communication networks are combined to obtain the important value of GIS in the management of communication networks [20]. Based on a comparison of various domestic and foreign existing and semi-experienced propagation models, each model is used for simulation practice. Finally, it is given that different propagation environments should choose different LTE propagation models [21]; the use of GIS technology to obtain the research area Building height, density and other information, collected a large amount of measured data, the prediction model 
Table 1 Classification of wireless communication signals by frequency

\begin{tabular}{|c|c|c|c|c|}
\hline Band name & Wavelength range & Frequency range & Propagation mode & Application occasion \\
\hline Long wave band & 2000-20000 m & $30-300 \mathrm{kHz}$ & Ground wave & $\begin{array}{l}\text { Long distance com- } \\
\text { munication }\end{array}$ \\
\hline Mid-band & 200-2000 m & $300-3000 \mathrm{kHz}$ & Ground wave & $\begin{array}{l}\text { Broadcast, communi- } \\
\text { cation, navigation }\end{array}$ \\
\hline Short wave band & $20-200 m$ & $3-30 \mathrm{MHz}$ & Sky wave & $\begin{array}{l}\text { Middle distance com- } \\
\text { munication }\end{array}$ \\
\hline $\begin{array}{l}\text { Ultra-short wave } \\
\text { band }\end{array}$ & I 20 m & $30-300 \mathrm{MHz}$ & $\begin{array}{l}\text { Linear propagation, } \\
\text { scattering }\end{array}$ & $\begin{array}{l}\text { Mobile communica- } \\
\text { tion, TV broadcasting }\end{array}$ \\
\hline $\begin{array}{l}\text { Decimeter wave } \\
\text { band }\end{array}$ & 200-2000 m & $300-3000 \mathrm{MHz}$ & $\begin{array}{l}\text { Linear propagation, } \\
\text { scattering }\end{array}$ & $\begin{array}{l}\text { Mobile communica- } \\
\text { tions, satellite com- } \\
\text { munications, }\end{array}$ \\
\hline $\begin{array}{l}\text { Centimeter wave } \\
\text { band }\end{array}$ & I 20 m & $3-30 \mathrm{GHz}$ & Straight propagation & TV broadcasting, radar \\
\hline Millimeter wave & I $20 \mathrm{~m}$ & $30-300 \mathrm{GHz}$ & Straight propagation & $\begin{array}{l}\text { Relay communication, } \\
\text { radar, satellite }\end{array}$ \\
\hline
\end{tabular}

obtained is consistent with the actual situation, and a specific method for predicting the coverage of wireless communication signals based on the building's field information is designed [22, 23].

According to the basic electromagnetic wave propagation theory in mobile communications, radio waves are affected by factors such as terrain, frequency, and distance. They propagate in the propagation space with path loss, slow fading loss, and fast fading. Multipaths are generated from two aspects, time and space loss, affected by terrain and features, will produce shadow effects, near and far effects, and Doppler effects. According to the electromagnetic wave space propagation attenuation theory, there are mainly theoretical analysis methods and field measurement methods for studying the electromagnetic field propagation space field distribution of mobile communication base stations. This paper mainly uses statistical prediction models to perform signal coverage prediction. After comparing the commonly used classic outdoor macro-cell signal propagation models, the propagation model suitable for the study area is determined to be the SPM general model. This model considers the signal propagation path loss and the distribution of ground features, antenna height, carrier frequency, distance, and other various effects of parameters on the signal. Then, the received level signal is predicted by the LTE downlink budget method, and a weighted least square method is used to fit a local prediction model, and the prediction result is verified to meet the index evaluation. The coverage of each TD-LTE base station in the study area was calculated, and the prediction results of the wireless signal propagation path loss coverage of each base station were obtained. Using GIS analysis tools and network planning software, a visual simulation of the loss prediction results was achieved.

\section{Methodology}

\subsection{Classification of wireless communication signals}

Table 1 details the classification of wireless communication signals by frequency. It included band name, wavelength range, frequency range, propagation mode, and application occasions. 


\subsection{TD-LTE communication data processing method}

(1) Data filtering.

It is generally considered that the range between $0.1 \mathrm{R}$ and $2 \mathrm{R}$ from the slave station is a reasonable test range, and $\mathrm{R}$ is the expected test cell radius. The strength of the test signal does not have a strict linear relationship with the propagation distance. The test distance is too close, the test data are small, and geographical averaging cannot be performed. At this time, the receiving terminal is in a critical state to resolve the signal, and its value is easily affected by transient fluctuations. In addition, some test data must be filtered from the sampling data. The specific filtering conditions are as follows:

1. Under the overhead, medium tunnel, where GPS cannot locate accurately.

2. Data that are too close or too far away from the antenna, and data that are too close or too far away are not easy to average.

3. Data with too weak signal strength (less than $-115 \mathrm{dBm}$ )

4. Incorrect data caused by inaccurate antenna pattern

5. Data on other sections that have been determined not to meet the requirements on the $\mathrm{CW}$ test route, such as: test data for repeated sections;

6. Signal strength exceeding one $40 \mathrm{dBm}$ but unexplainable fading;

Filters of types 1 and 5 need to be completed before geographic averaging, and filters of types 2 and 3.4 need to be completed after geographic averaging of data.

(2) Data discrete processing principle.

Assuming that the vehicle speed between the two data collection points is uniform, and the time difference between each two recording points is equal, the receiver data sampling speed is much faster than the GPS positioning speed. The test records cannot be directly geographically averaged; usually, these collected data are evenly distributed to the road section between the two test points in chronological order to meet a sufficient number of points per $6 \mathrm{~m}$ in length.

(3) Geographic average.

The purpose of geographic averaging is to eliminate fast fading and retain the effects of slow fading. There are two methods of geographic averaging:

Method 1: The entire test area is made into a grid with a side length of $6 \mathrm{~m}$, and the communication data collected in each grid are arithmetically averaged.

Method two: Separately spaced along the test route, $6 \mathrm{~m}$ per segment, arithmetically average the test data collected in each segment, and uniformly select a point as the average test point.

\subsection{Analysis of wireless communication network propagation model}

The GIS-based wireless communication network propagation model uses topographic data, that is, a digital electronic map database, multiple diffraction mechanisms, taking into account the propagation conditions in different landscapes, and effective antenna heights. At the same time, the model can be corrected. The model is based on the following formula: 


$$
\begin{aligned}
\mathrm{PR}= & \mathrm{PIX} \\
& -\left\{\begin{array}{l}
K 1+K 2 \lg (d)+K 3 \lg (\text { HIXeff })+K 4 * \text { Diffraction }+K 5 \lg (d) * \lg (\text { HIXeff }) \\
+K 6(\text { HRXeff })+K c l u t t e r
\end{array} * f(\text { clutter })\right.
\end{aligned}
$$

The parameters in the formula are as follows:

PR: terminal received power $(\mathrm{dBm})$.

PTx: Base Station Transmit Power (EIRP) (dBm).

K1: Frequency-dependent constant (dB).

K2: Multiplier factor for $\operatorname{Lg}(d)$. This value indicates how fast the field strength changes with distance.

$\mathrm{d}$ : distance between transmitter and receiver $(\mathrm{m})$.

K3: $\lg$ (HTxeff) multiplier factor, this value indicates the field strength changes with the antenna height.

HTXeff: effective height of the transmitting antenna $(\mathrm{m})$.

K4: multiplier factor for diffraction calculation, K4 must be a positive number.

Diffraction: Diffraction loss $(\mathrm{dB})$ caused by passing an obstacle path.

K5: Multiplier for $\operatorname{Lg}$ (TXHeff) and $\operatorname{Lg}(d)$.

K6: HRxeff multiplier.

Hrxeff: effective height of the receiving antenna (m).

Kclutter: multiplier factor for $f$ (clutter), this value represents the weight of ground loss.

$f$ (clutter): average weighted loss of different features.

It is worth noting that the model calculates the loss $f$ (clutter) caused by the ground features, which is a weight loss due to the ground features passing from the transmitter to the receiver. The weighting method is also optional.

\subsection{Optimization of wireless communication network propagation model}

The method of using this model to correct is: first set the parameter values K1-K6, usually you can choose the default value of the frequency to set it, or other similar terrain correction parameters, and then use this model for wireless propagation prediction. Compare the predicted value with the drive test data and then modify the model parameters based on the statistical results of the obtained difference. After continuous iterative processing, until the mean square error and standard deviation of the top test value and the drive test data reach the minimum, each parameter value of the model obtained at that time is the required correction value.

The principle used in the above method is the principle of least squares, and the theory is as follows:

Let a plane straight line (i.e., one-variable regression) fit. Let the theoretical straight line be $y=a+b x$. There are $\mathrm{n}$ sets of measured values $(x l, y l),(\times 2, y 2) \ldots(x n, y n)$, which is nearly linearly distributed in plane coordinates.

The principle of the least square method is to say that if the sum of squares of the difference between the theoretical positions of the actual measured values $\mathrm{t} 7$ is the smallest, then this theoretical straight line is the regression that best reflects the actual measured values. 


$$
\Delta=\sum_{i=1}^{n}\left(y_{i}-y\right)^{2}=\sum_{i=1}^{n}\left(y_{i}-a-b x_{i}\right)
$$

Finding regression coefficients $a$ and $b$ by partial derivative method.

$$
\frac{\partial \Delta}{\partial a}=2 \sum_{i=1}^{n}\left(y_{i}-a-b x_{i}\right)=0
$$

which is:

$$
\begin{aligned}
& \sum_{i=1}^{n} y_{i}=n a+b \sum_{i=1}^{n} x_{i} \\
& \frac{\partial \Delta}{\partial b}=2 \sum_{i=1}^{n}\left(y_{i}-a-b x_{i}\right)=0 \\
& \sum_{i=1}^{n} x_{i} y_{i}=a \sum_{i=1}^{n} x_{i}+b \sum_{i=1}^{n} x_{i}^{2}
\end{aligned}
$$

Find the regression coefficients $a$ and $b$.

Let $x 1$ be the predicted value of a test point, $x 2$ be the measured value at that point, $y_{i}=x 1-x 2, \bar{y}=\frac{\sum y_{i}}{N}$ and $\bar{y}$ be the statistical average difference

$$
\begin{aligned}
\text { RMS,Error } & =\sqrt{\frac{\sum y_{i}^{2}}{N}} \\
\text { StdError } & =\sqrt{\frac{\sum\left(y_{i}-\bar{y}\right)^{2}}{N-1}}
\end{aligned}
$$

It can be seen that when $\bar{y}$ is 0 , RMS Error $=$ StdError.

The result of model optimization is to make the values of RMS Error and StdError to a minimum, and to judge the fit between the model results and the actual environment. The parameter correction algorithm for the SPM model is as follows:

First define the following parameters: $L$ is the theoretical loss value; $L 1$ is the actual loss value; $D L=L 1-L$ is the difference between the actual value and the theoretical value; $K 1$ is a correction factor related to frequency $f: K 2$ : based on $\log (d)$ Correction factor. After sampling analysis of the system, a matrix $\log (d)$ of the distance $d$ between the transmitter and the receiver is obtained.

$$
\lg d n=(\lg d 1, \lg d 2 \ldots \lg d n)
$$

Difference between theoretical value and actual value $\triangle \mathrm{Ln}$.

$$
\triangle \operatorname{Ln}=(\triangle L 1, \triangle L 2 \ldots \Delta \operatorname{Ln})
$$

Define the correction factor constant: $K=(K 1, K 2)$

$$
K=\triangle \operatorname{Ln} / A
$$


Table 2 K value correction coefficient term of SPM model

\begin{tabular}{llc}
\hline Kvalue & Coefficient meaning & $\begin{array}{l}\text { Whether } \\
\text { to } \\
\text { correct }\end{array}$ \\
\hline$K 1$ & Constant loss, frequency dependent & $\mathrm{Y}$ \\
$K 2$ & Transmission distance loss & $\mathrm{Y}$ \\
$K 3$ & Loss factor caused by changes in base station antenna height & $\mathrm{N}$ \\
$K 4$ & Diffraction loss, only hilly terrain will be considered & $\mathrm{N}$ \\
$K 5$ & Influence of base station antenna height and propagation distance on received power & $\mathrm{N}$ \\
$K 6$ & $\quad$ Gain caused by changes in antenna height at the receiving end, when the terminal & $\mathrm{N}$ \\
& $\quad$ height is 1.5 m, usually set to 0 & $\mathrm{N}$ \\
Kclutter & Various ground loss, the default value is 1 &
\end{tabular}

\section{Optimization and visualization of TD-LTE wireless communication network model based on GIS}

\subsection{SPM propagation model optimization algorithm}

Although theoretically all $K$ parameters of the SPM model can be corrected, in practical applications, due to various conditions during data collection, the experimental data are limited, so not all $K$ coefficients can be accurately corrected. Generally speaking, the propagation distance is an important factor affecting the path loss, so correction of the coefficient $K$ of lgd is a priority. In addition, the carrier frequency has a certain effect on the loss result, so the frequency factor $K 1$ must also be corrected. In addition to the above two coefficients, other K-factor correction suggestions are shown in Table 2.

Since the antenna height of each base station remains unchanged during the data acquisition process, the degree of change in the height of the base station antenna is a coefficient $K 3$. Because the change in terrain is generally not obvious, the antenna height is considered to be unchanged, so it is recommended that $K$ : take the default value. No corrections are made. Because the area under study is flat in the suburbs or rural areas, and the difference is determined, it is not recommended to correct $K 4$. The correlation coefficient $K 5$ of the antenna height of the base station and the signal propagation distance is not corrected. Similar to $K 3$, it is also recommended to take the default value without correction.

The formula of the TD-LTE path loss model is:

$$
\begin{aligned}
L_{\text {pathloss }} & =K 1+K 2 \lg d+K 3 \lg \left(h_{t e}\right)+K 5 \lg h_{t e} \lg d \\
& =K 1+K 3 \lg \left(h_{t e}\right)+\left(K 5 \lg h_{t e}+K 2\right) \lg d
\end{aligned}
$$

Among them, $K 2$ takes a typical value of $5.83, K 5$ takes a typical value -6.55 .

The purpose of parameter correction is to use the measured data to fit a linear regression equation, and finally list the regression equation to calculate the $K$ parameter. The above formula is organized as:

$$
\begin{aligned}
L_{\text {pathloss }} & =K 1+K 3 \lg \left(h_{t e}\right)+\left(K 2+K 5 \lg h_{t e}\right) \lg d \\
& =a+b \lg d \\
& =a+b x
\end{aligned}
$$




\subsection{SPM propagation model optimization process}

(1) LTE outdoor downlink budget method.

The focus is on the path loss when the TD-LTE system signals are propagated in the downlink, that is, the downlink budget. Downlink loss formula:

$$
L_{\mathrm{RSRP}}=P_{t e}-L_{f}+G_{t e}+G_{r e}-M_{f}-M_{I}-L_{P}-L_{b}-S_{u e}
$$

Except for the factors that cause the signal power to change due to the LTE system settings, the losses caused by radio waves during space propagation are collectively referred to as path loss $L_{\text {pathloss, }}$, so the above link formula is converted into:

$$
L_{\mathrm{RSRP}}=P_{t e}-L_{f}+G_{t e}+G_{r e}-L_{\text {pathloss }}
$$

According to the actual measured base station data, where: $L_{f}=6 \mathrm{dBm}, G_{t e}=8 \mathrm{dBm}, G_{r e}=0$, the measured path loss:

$$
L_{\text {pathloss }}=-L_{\mathrm{RSRP}}+P_{t e}+2
$$

The base station transmit power $P_{t e}$ depends on the actual parameters of the base station; the mobile station height is $1.5 \mathrm{~m}$; distance: the distance is calculated. According to the GPS geographical coordinates of each sampling point, the straight line distance from each base station in the serving cell can be calculated, and finally the distance the logarithmic value of $\lg d$ (the unit of $\mathrm{d}$ is $\mathrm{m}$ ). The nature of the model correction is to fit a regression curve that conforms to the actual value, to obtain the corrected model coefficient, and to substitute the corrected model coefficient into the calculated predicted value. The error should be minimized. At present, the commonly used algorithm for model correction is the least square method, which is also commonly used by network planning simulation software.

The weighted least squares method is to weight the original basic model to make it a new model without heteroscedasticity, that is, to give each data a different weighting value $\omega_{i}$ when the data are fitted and then use the ordinary minimum. The square method estimates its parameters so that the sum of the squares of the deviations between the measured and predicted values reaches a minimum.

Let the plane be fitted with a straight line. The theoretical straight line is $Y=a+b x$. There are $n$ sets of measured data $\left(x_{i}, y_{i}\right)(i=1,2, \ldots, n)$ that are linearly distributed on the plane. The expression of the weighted least squares method is as follows:

$$
J=\sum_{i=1}^{n} \omega_{i} \delta_{i}^{2}=\sum_{i=1}^{n} \omega_{i}\left[y_{i}-\overline{y_{i}}\right]^{2}
$$

Among them, $J$ represents the weighted sum of squared deviations, $y_{i}$ is the actual value, and $\overline{y_{i}}$ is the predicted value. The values of the coefficients $a$ and $b$ are obtained using the Lagrange multiplier method.

\subsection{Visualization of wireless communication propagation prediction based on GIS}

Combining GIS technology with mobile communication signal propagation technology, performing path loss calculation of signals transmitted by communication 


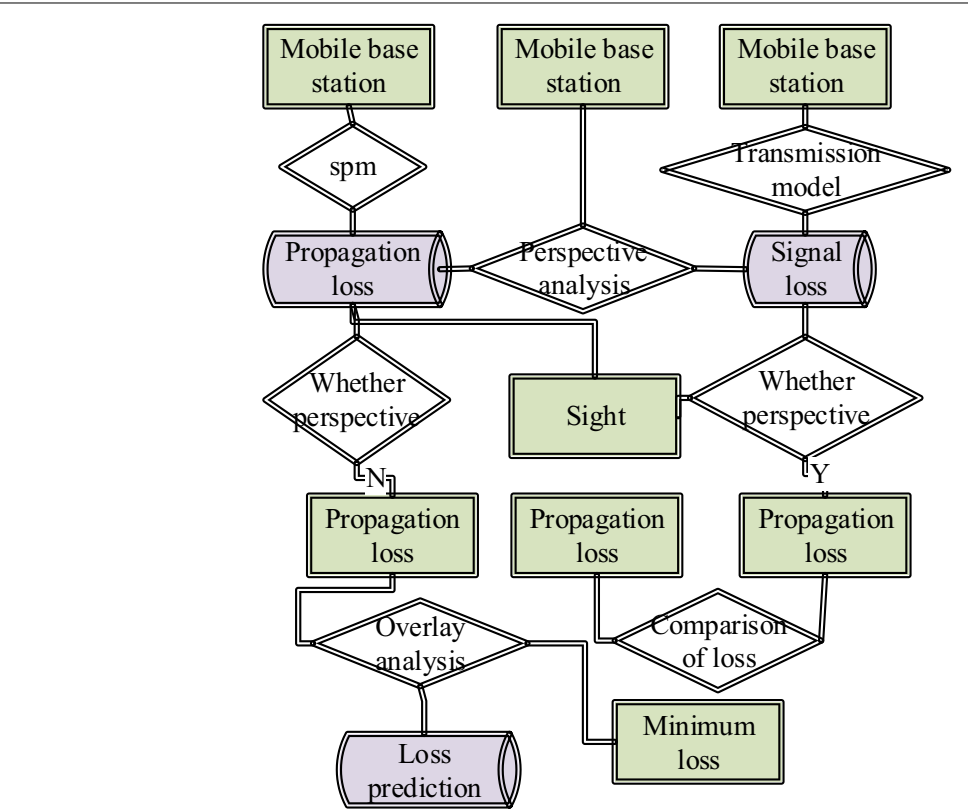

Fig. 1 Visual simulation flow of wireless communication network propagation loss prediction

base stations in the study area, and finally obtaining point-by-point signal coverage and signal strength in the study area, thereby realizing visualization and prediction of mobile communication signal propagation and simulation. Specific visualization methods, using ArcGIS spatial analysis tools, based on the GIS model to calculate the communication signal coverage and signal intensity of each point in the study area; when restricted by the regional terrain conditions, the calculation conditions of this model cannot be applied, the application of visual field analysis and electromagnetic waves. The free space transmission loss formula supplements the area coverage, that is, the electromagnetic wave free space propagation loss formula is used to calculate the communication loss in the base station point's passable sight. The specific flowchart is shown in Fig. 1.

Create a point feature SHP file. Based on the actual geographic coordinates of each base station, the data of 10 experimental base stations were imported into ARCGIS and the base station layer was formed with the DEM topographic map superimposed on the building height, as shown in Fig. 2 below (Table 3).

The field of view analysis should be combined with the parameters of the base station, such as the height of the base station, the azimuth of the base station, the mechanical downtilt of the base station, and the coverage radius of the base station. Therefore, according to actual needs, add some fields to the attribute table, some of the data are shown in Table 4 below.

\section{Results and discussion}

The single-length path of the measured data is about $23 \mathrm{~km}$, but in order to obtain more data, each base station repeats the test 4 times. The measured data points are 16,144. The segmented statistics of RSRP level signal values are shown in Table 5, where the 


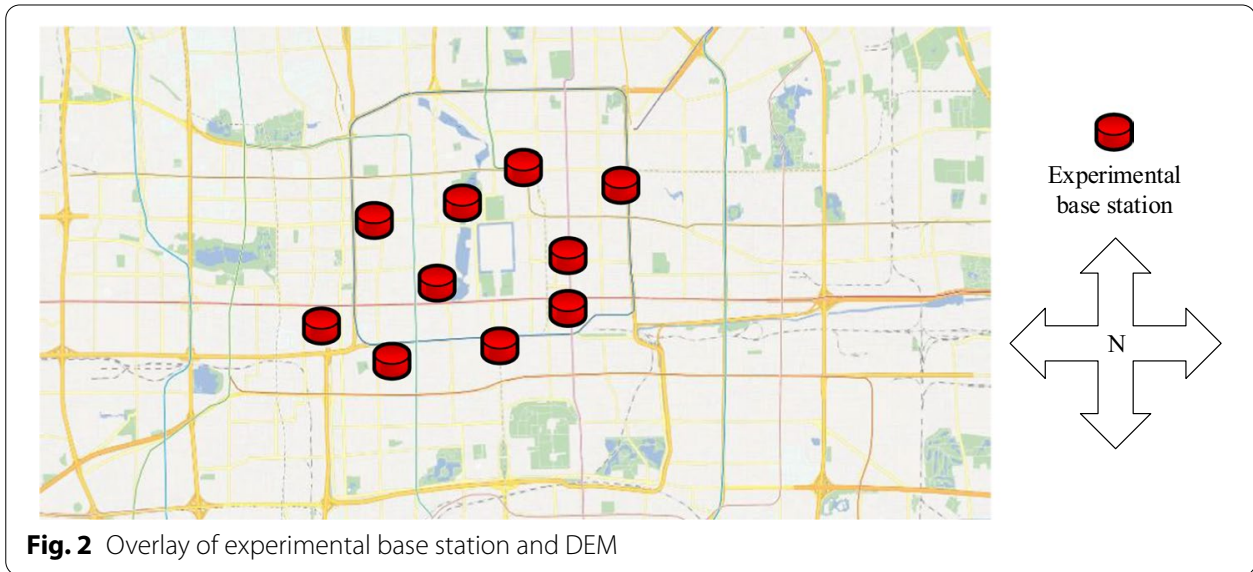

Table 3 Meaning of each parameter of the downlink

\begin{tabular}{|c|c|c|}
\hline Parameter & Meaning & Default value \\
\hline Body loss & Loss when signal penetrates human body & $0 \mathrm{~dB}$ \\
\hline UE antenna gain & Receiver antenna gain & $0 \mathrm{dBi}$ \\
\hline Base station antenna gain & Transmit antenna gain & $18 \mathrm{dBi}$ \\
\hline Feeder loss & $\begin{array}{l}\text { Loss of all cables and connectors from the set top to the transmit- } \\
\text { ting antenna }\end{array}$ & $1-4 \mathrm{~dB}$ \\
\hline Penetration loss & Signal loss through buildings, cars, ships, etc & $10-20 \mathrm{~dB}$ \\
\hline Shadow fade margin & $\begin{array}{l}\text { A method to allow for a certain margin considering the effects of } \\
\text { shadow effects }\end{array}$ & - \\
\hline Receiver sensitivity & Lowest level to ensure the receiver can work normally & $-106 \mathrm{dBm}$ \\
\hline Interference margin & $\begin{array}{l}\text { In the actual propagation environment, the signal of the mobile } \\
\text { receiver will be affected by its Interference of other signals, the } \\
\text { margin value reserved for this purpose is the interference margin } \\
\text { the amount }\end{array}$ & - \\
\hline Base station transmit power & Transmitting antenna power & $40 \mathrm{dBm}$ \\
\hline
\end{tabular}

Table 4 Fields added in the base station of the visible domain

\begin{tabular}{llrrl}
\hline OFFSETA & VERT2 & AZIMUTH1 & AZIMUTH2 & RADIUS2 \\
\hline 15 & 13 & 0 & 65 & 2000 \\
15 & 12 & 60 & 256 & 2000 \\
16 & 12 & 252 & 345 & 2000 \\
24 & 13 & 0 & 35 & 2000 \\
25 & 6 & 32 & 165 & 2000 \\
25 & 8 & 162 & 270 & 2000 \\
27 & 13.78 & 0 & 25 & 2000 \\
\hline
\end{tabular}

minimum value is $-109 \mathrm{dBm}$, the maximum value is $-52 \mathrm{dBm}$, and the average value is $-78 \mathrm{dBm}$.

According to the $K$ parameter correction, $K 3=5.83, K 5=-6.55$ take the default values to correct $K 1, K 2$. After the SPM model is corrected and the data are preprocessed, the weighted least squares method is used to calculate the linear regression equations of the 3 base stations as shown in Fig. 3 below: 
Table 5 RSRP level signal segment statistics

\begin{tabular}{lllll}
\hline RSRP & & & & \\
\hline Order & Range & Samples & PDF & CDF \\
\hline 1 & {$[-$ inf, -130$]$} & 0 & $0 \%$ & $0 \%$ \\
2 & {$[-130,-105]$} & 16 & $0.2 \%$ & $0.15 \%$ \\
3 & {$[-105,-90]$} & 512 & $4.17 \%$ & $3.29 \%$ \\
4 & {$[-90,-80]$} & 5233 & $32.34 \%$ & $35.89 \%$ \\
5 & {$[-80,-76]$} & 7089 & $43.8 \%$ & $79.68 \%$ \\
6 & {$[-76$, inf $]$} & 3297 & $20.5 \%$ & $100 \%$ \\
Total & 16,133 & & Average & -68.97 \\
Maximum & -53 & & Minimum & -108 \\
\hline
\end{tabular}

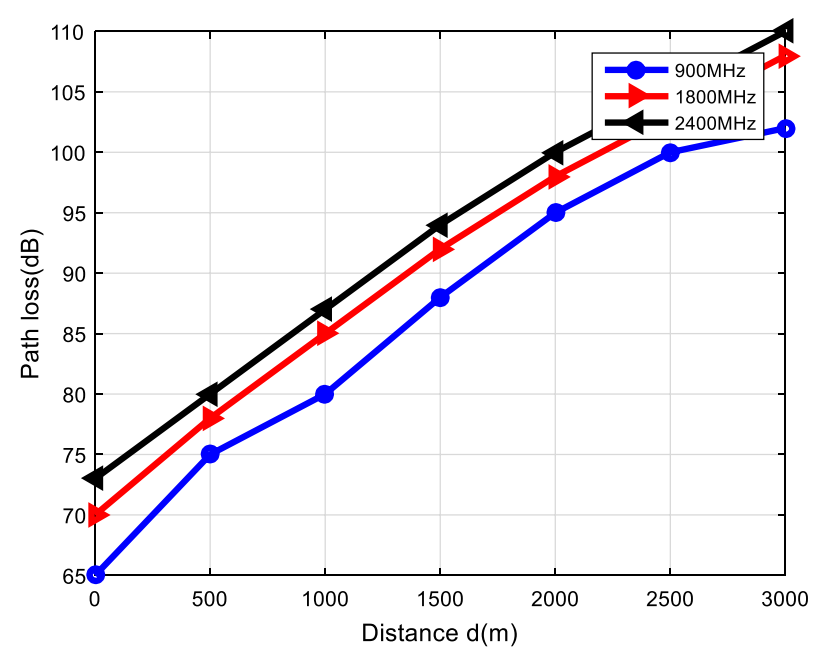

Fig. 3 Comparison of the propagation loss of the linear regression correction model of each base station

The effective height of the test base station antenna is known. Substituting the $a$ and $b$ values of the regression equation into the formula, the $K 1$ and $K 2$ values of the test base stations are shown in Table 6:

This paper uses the weighted least squares method to analyze the linear regression equation and obtain the prediction improvement model. However, after the model correction is completed, it is necessary to verify whether the model meets the engineering requirements. The actual loss fitting effect after the correction of each base station is shown in Fig. 4.

TD-LTE network wireless signal effective flow product is 20.38 square kilometers, within this effective range, 298 distribution network communication points were selected for signal strength testing. The test results are shown in Fig. 5. The test results show that $79 \%$ of the distribution points have good signals (RSRP $>-115 \mathrm{dBm}$ ) and $15 \%$ have poor signals $(-115 \mathrm{dBm}<\mathrm{RSRP}>-125 \mathrm{dBm})$.

As above, the weighted least squares method was used to correct the SPM models of the base stations, and an improved model applicable to each base station was obtained. 
Table 6 Values of the test base stations

\begin{tabular}{lll}
\hline Base station & K1 & K2 \\
\hline Yihao & 33.67 & 43.72 \\
Shenlian Electronics & 33.76 & 43.11 \\
Tong He Restaurant & 36.54 & 42.07 \\
Hele New Village & 48.91 & 38.95 \\
Jingji Power Station Trailer & 38.25 & 42.98 \\
Rongde Nonferrous New Materials & 41.61 & 41.26 \\
East District of Hele New Village & 34.26 & 46.56 \\
Dormitory building of Zhongshenglong Electronics Co., Ltd & 28.33 & 44.69 \\
Zhongshenglong Electronics Co., Ltd & 27.38 & 46.1 \\
River Tungsten Tyco Landscape Tower & 29.25 & 45.01 \\
Beyond Technology Landscape Tower & 34.42 & 42.44 \\
Yellow Sand D & 28.89 & 43.91 \\
Hele New Village (Hualong Mobile Supermarket) & 53.68 & 37.61
\end{tabular}

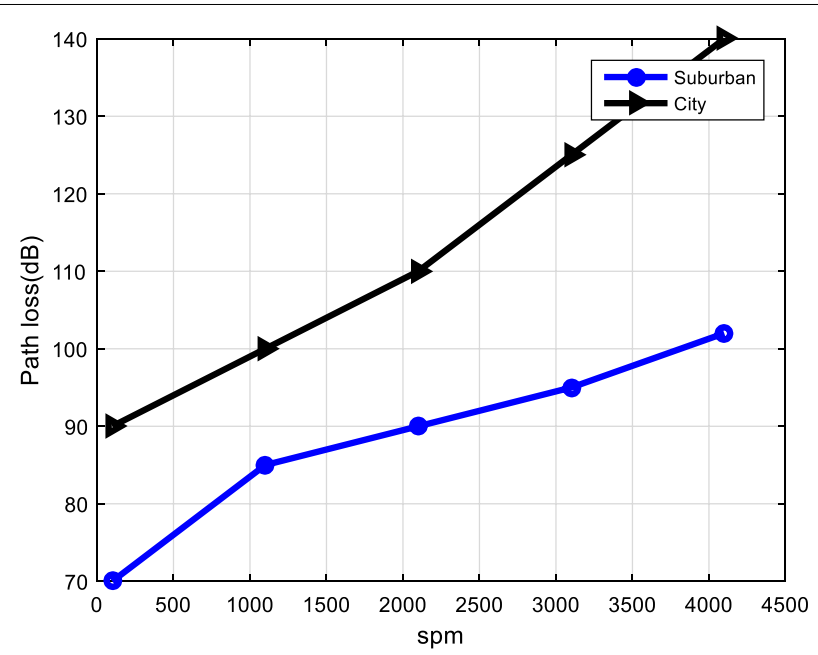

Fig. 4 Actual loss prediction chart

The corrected $K 1, K$ : parameters are within the reference range and meet the model requirements. The final parameter verification results show that the average and variance of the actual and predicted values meet the evaluation requirements, and the model correction results are good.

\section{Conclusion}

The key technologies of each part of the TD-LTE wireless network planning process are studied. Including the determination of planning goals, the analysis of propagation models, the estimation of coverage and capacity, the analysis and design of wireless network parameters, and the design of the simulation process, the focus of which 


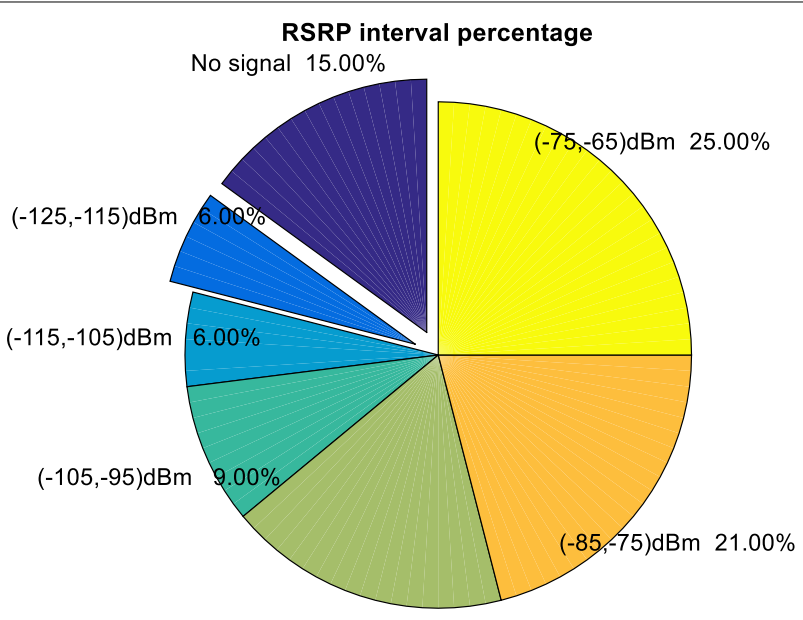

$(-95,-85) \mathrm{dBm} \quad 18.00 \%$

Fig. 5 RSRP values of 298 distribution points in the TD-LTE signal coverage area

is the estimation of coverage and capacity, the TD-LTE wireless network planning is constructed in detail Coverage and capacity estimation. According to the theoretical analysis, the general algorithm and basic procedure of model correction are designed, and the K parameters suitable for the TD-LTE system in the study area are obtained by fitting. The result is that the propagation prediction model improved by this method is close to the measured data and has a certain practical reference value.

\section{Abbreviations}

GIS: Geographic Information System; SPM: Standard Propagation Model; TD-LTE: Time Division Long-Term Evolution; GSM: Global System for Mobile Communications; CDMA: Code Division Multiple Access; LTE: Long-Term Evolution; GPS: Global Positioning System; RMS: Root Mean Square; RSPR: Reference Signal Receiving Power.

\section{Acknowledgements}

The author would like to express her sincere thanks to the referees for the careful reading and their important comments which helped improve the original paper.

\section{Funding}

Not applicable.

Availability of data and materials

Not applicable.

\section{Declaration}

\section{Competing interests}

The authors declare that they have no competing interests.

\section{Author details}

${ }^{1}$ School of Economics and Management, Nanjing Polytechnic Institute, Nanjing, China. ${ }^{2}$ Business School, Jinling Institute of Technology, Nanjing, China. ${ }^{3}$ School of Business Administration, Hubei University of Economic, Wuhan, China.

Received: 13 April 2021 Accepted: 23 May 2021

Published online: 31 July 2021

\section{References}

1. L. Ma, S. Hu, Q. Min et al., Energy consumption optimization of high sulfur natural gas purification plant based on back propagation neural network and genetic algorithms. Energy Procedia 105, 5166-5171 (2017)

2. D. Djomadji, T. Emmanuel, New propagation model optimization approach based on particles swarm optimization algorithm. Int. J. Comput. Appl. 118(10), 975-8887 (2015) 
3. T. Li, S. Ying, P. He, Research on the material mechanics performance prediction based on back propagation neural network. J. Comput. Theor. Nanosci. 13(4), 2519-2523 (2016)

4. J. Wei, X. Yu, Y. Wang, The optimization model based on traffic flow and safety. J. Comput. Theor. Nanosci. 12(12), 6265-6271 (2015)

5. Q. Liu, X. Wan, D. Xie, Optimization of spectral printer modeling based on a modified cellular Yule-Nielsen spectral Neugebauer model. J. Opt. Soc. Am. A 31(6), 1284-1294 (2014)

6. Z.K. Adeyemo, O.K. Ogunremi, I.A. Ojedokun, Optimization of Okumura-Hata model for long term evolution network deployment in lagos, nigeria. Int. J. Commun. Antenna Propag. 6(3), 146 (2016)

7. J.H. Pan, X. Liu, Assessment of landscape ecological security and optimization of landscape pattern based on spatial principal component analysis and resistance model in arid inland area: a case study of Ganzhou District, Zhangye City, Northwest China. Ying Yong Sheng Tai Xue Bao 26(10), 3126-3136 (2015)

8. X. Yang, G. Xu, L. Qi et al., Authorship attribution of source code by using back propagation neural network based on particle swarm optimization. PLoS ONE 12(11), 187-204 (2017)

9. R.J.M. Geelen, Y. Liu, J.E. Dolbow, An optimization-based phase-field method for continuous-discontinuous crack propagation. Int. J. Numer. Methods Eng. 116(1), 345-367 (2018)

10. Y. Ye, K. Sun, L. Kuang, Spatial layout optimization of urban space and agricultural space based on spatial decisionmaking. Trans. Chin. Soc. Agric. Eng. 33(16), 256-266 (2017)

11. F. Gu, Y.X. Huang, C.M. Chen, Construction and optimization of ecological network for nature reserves in Fujian Province, China. Chin. J. Appl. Ecol. 28(3), 1013-1020 (2017)

12. K.N. Sayl, N.S. Muhammad, A. El-Shafie, Optimization of area-volume-elevation curve using GIS-SRTM method for rainwater harvesting in arid areas. Environ. Earth Sci. 76(10), 368-378 (2017)

13. C. Hou, Y. Xiao, Y. Cao et al., Prediction of synchronous closing time of permanent magnetic actuator for vacuum circuit breaker based on PSO-BP. IEEE Trans. Dielectr. Electr. Insul. 24(6), 3321-3326 (2018)

14. A. De La Fuente, G. Femenias, F. Riera-Palou, Subband CQI feedback-based multicast resource allocation in MIMOOFDMA networks. IEEE Trans. Broadcast. 64(4), 846-864 (2018)

15. J. Palacios, D. De Donno, J. Widmer, Lightweight and effective sector beam pattern synthesis with uniform linear antenna arrays. IEEE Antennas Wirel. Propag. Lett. 16(99), 605-608 (2017)

16. G. Zuccaro, D. De Gregorio, M. Titirla, On the simulation of the seismic energy transmission mechanisms. Ing. Sismica 35(1), 567-578 (2018)

17. N. Wang, M. He, H. Wang, Underwater target feature extraction method based on convolutional neural network. Xi Tong Gong Cheng Yu Dian Zi Ji Shu/Syst. Eng. Electron. 40(6), 1197-1203 (2018)

18. J. Du, C. Roblin, Stochastic surrogate models of deformable antennas based on vector spherical harmonics and polynomial chaos expansions: application to textile antennas. IEEE Trans. Antennas Propag. 66(7), 3610-3622 (2018)

19. M.S. Hossain Lipu, M.A. Hannan, A. Hussain, State of charge estimation for lithium-ion battery using recurrent NARX neural network model based lighting search algorithm. IEEE Access 6(2018), 28150-28161 (2018)

20. S. Wang, Z. Hu, C. Rong, Optimization analysis of coil configuration and circuit model for asymmetric wireless power transfer system. IET Microwaves Antennas Propag. 12(7), 1132-1139 (2018)

21. S. Shen, C.Y. Chiu, R.D. Murch, Multiport pixel rectenna for ambient RF energy harvesting. IEEE Trans. Antennas Propag. 66(2), 644-656 (2018)

22. X. Sun, J. Kong, Z. Liu, Middle-term power load forecasting model based on kernel principal component analysis and improved neural network. Nanjing Li Gong Daxue Xuebao/J. Nanjing Univ. Sci. Technol. 42(3), 259-265 (2018)

23. Y.-B. Wang, D.-G. Chang, Y.-H. Fan, Acoustic localization of partial discharge sources in power transformers using a particle-swarm-optimization-route-searching algorithm. IEEE Trans. Dielectr. Electr. Insul. 24(6), 3647-3656 (2017)

\section{Publisher's Note}

Springer Nature remains neutral with regard to jurisdictional claims in published maps and institutional affiliations.

\section{Submit your manuscript to a SpringerOpen ${ }^{\circ}$ journal and benefit from:}

- Convenient online submission

- Rigorous peer review

- Open access: articles freely available online

- High visibility within the field

- Retaining the copyright to your article

Submit your next manuscript at $\gg$ springeropen.com 